\title{
Escuelas secundarias parroquiales en barrios precarios: Segregación residencial e inclusión educativa
}

\author{
Parochial secondary schools in precarious neighborhoods: \\ Residential segregation and educational inclusion
}

\author{
Mariana Cecilia Facciola ${ }^{1}$ \\ Ana Lourdes Suárez ${ }^{2}$
}

\begin{abstract}
Resumen: Se presentan los resultados de un estudio de caso en dos escuelas parroquiales de barrios precarios de Buenos Aires cuyo objetivo fue analizar la interrelación entre segregación residencial, segmentación educativa e inclusión socioeducativa. Los datos se relevaron a través de un cuestionario sobre el perfil sociodemográfico y otro sobre el axiológico de los alumnos; se recurrió asimismo a entrevistas a directivos y a los proyectos educativos de ambas escuelas. El análisis de los datos evidenció vulnerabilidad socioeconómica de los alumnos agravada por habitar en espacios segregados de la ciudad y la participación en circuitos escolares de baja calidad. Se muestra asimismo que las visiones, motivaciones y propuestas educativas de ambas escuelas constituyen un capital cultural muy relevante para los estudiantes quienes, según los datos del cuestionario axiológico, manifiestan dos dimensiones descendidas: reconocimiento social y liderazgo. Se concluye en la necesidad de un ajuste curricular situado y focalizado en los alumnos.
\end{abstract}

Palabras clave: segregación, educación, aprendizaje, escolaridad, sistema de valores.

\footnotetext{
${ }^{1}$ Doctora en Psicopedagogía. Docente de la Universidad Católica Argentina. Directora del Departamento de Psicopedagogía de la Universidad Católica Argentina. Buenos Aires, Argentina.

E-mail: mariana_facciola@uca.edu.ar.

2 Doctora en Sociología. Investigadora independiente de CONICET. Docente e investigadora de la Universidad Católica Argentina. Buenos Aires, Argentina. E-mail: analourdessuarez@gmail.com.
}

Diálogos Pedagágicos. ISSN en línea: 2524-9274

Año XVI, No 32, octubre 2018-marzo 2019. Pág. 72-94

DOI: http://dx.doi.org/10.22529/dp.2018.16(32)05 / Recibido:15-06-2018 / Aprobado: 13-08-2018

cc)(-) $\Theta$ Artículo publicado bajo Licencia Creative Commons Atribución-NoComercial-SinDerivar cc. Universidad Católica de Córdoba. 


\begin{abstract}
The article presents result of a case study conducted in two parochial schools of Buenos Aires poor neighborhoods. The aim was to analyze the relationship between residential segregation, educational fragmentation, and socio-educational inclusion Two questionnaires and several interviews provided data for the study. From the sociodemographic profile of students there is evidence of social and economic vulnerability, worsened by living in segregated spaces, and the participation in low quality school segments. The axiological profile evidences two-dimensional very low: recognition and social leadership. Visions, motivations and educational proposals are important spiritual and cultural capital of both schools. From this case study we conclude on the need of a redesigned curriculum situated and focused on the territory.
\end{abstract}

Key words: segregation, education, learning, scholarship, value system.

\title{
Introducción
}

Las villas de emergencia ${ }^{3}$ de la ciudad de Buenos Aires (CABA) son espacios dinámicos por su acelerado crecimiento poblacional, por la diversidad cultural de su población, y por la lucha cotidiana de sus residentes en mejorar las condiciones de vida que acorten las brechas con el resto de la ciudad. Comenzaron a conformarse a mediados del siglo XX, y su persistente presencia evidencia la fragmentación y segregación residencial de Buenos Aires -la ciudad más poblada y rica del país-. La fuerte concentración de pobreza y de déficits urbanos permite afirmar que se trata de enclaves que comprueban el fracaso de las intervenciones ideadas para revertir los mecanismos de exclusión.

La presencia de agentes católicos -sacerdotes, religiosos/as y laicos/as- ha sido constante desde que comenzaron a conformarse estos territorios a mediados del siglo pasado, a través de un modo de estar, acompañar y desplegar una serie de actividades. ${ }^{4}$ Esta presencia motivó la reciente creación de establecimientos educativos para la finalización de nivel secundario en algunas de las villas. Esta iniciativa se enmarcó en un proyecto de presencia y acompañamiento a los jóvenes más vulnerables a través de una red de instituciones, como el colegio, la capilla y el club, porque hay que "confíar en la potencialidad de los nuestros"

\footnotetext{
3 Villas de emergencia es el nombre que reciben en Argentina las urbanizaciones muy precarias producidas por los mismos sectores populares en su necesidad de producirse un hábitat. Suelen ser barrios a los cuales se accede por medio de pasillos estrechos y tienden a crecer en altura ya que la disponibilidad de suelo es escasa o nula (Techo Argentina, 2013). De acuerdo a la información oficial se contabilizan en CABA 15 villas, 2 núcleos habitacionales transitorios y 24 asentamientos. Según el Censo Nacional de Población de 2010 residen en las villas unas 163.587 personas, lo que representa el $5,7 \%$ de la población total de la Ciudad en ese año (Suárez, Mitchell \& Lépore, 2014).

${ }^{4}$ La regular presencia de agentes religiosos en las villas de CABA se fortalece aún más con la creación de la Vicaría para la Pastoral en Villas de Emergencias en la Arquidiócesis en agosto del 2009, por decisión de Mons. Bergoglio -hoy Papa Francisco- entonces arzobispo de Buenos Aires.
} 
(Carrara, 2017, p. 9). ${ }^{5}$ Se impulsa así la presencia de estas tres instituciones sostenidas por la iglesia católica local como redes de contención que favorecen la pertenencia y configuran la identidad.

La escuela secundaria en contextos de marginalidad constituye un escenario de aprendizaje y adquisición de valores en la construcción de subjetividades; por lo que su trabajo con poblaciones muy vulnerables adquiere particular relevancia. Las escuelas parroquiales recientemente creadas en las villas de emergencia -objeto de nuestro estudio- reciben a alumnos de los estratos sociales más bajos y están ubicadas en espacios segregados residencialmente, por lo que enfrentan peculiares desafíos en relación con la inclusión, la calidad y la equidad. Romper con los circuitos de acumulación de desventajas del alumnado que reciben y ubicarse en segmentos educativos de alta calidad (conceptos que discutiremos en el próximo apartado) desafían fuertemente al proyecto pedagógico de estas escuelas.

La problemática descripta suscitó los interrogantes que han guiado el trabajo empírico y la reflexión interdisciplinaria: ${ }^{6}$ ¿cuál es el perfil sociodemográfico y la trayectoria educativa de los alumnos que asisten a establecimientos de nivel medio emplazados en los territorios más marginales de Buenos Aires?, ¿cuál es su perfil axiológico?, ¿cuál es la propuesta/respuesta pedagógica de las escuelas frente a estos perfiles?, ¿qué les demanda/implica a estas escuelas impartir educación de calidad y garantizar la inclusión social de sus alumnos? Estos interrogantes dieron forma a los objetivos de la investigación: 1. Confrontar el perfil sociodemográfico de todos los alumnos concurrentes a las dos escuelas parroquiales ubicadas en los asentamientos precarios, con la población comunal ${ }^{7}$ (territorial) donde están emplazas las escuelas y del total de la ciudad de Buenos Aires. 2. Visibilizar los perfiles axiológicos de los estudiantes que asisten a las escuelas secundarias parroquiales seleccionadas. 3. Identificar los rasgos significativos de las propuestas educativas de las escuelas parroquiales para la población de estudiantes en riesgo educativo. 4. Analizar la adecuación de la propuesta educativa al perfil de los alumnos garantizando educación inclusiva y de calidad.

\section{Referentes conceptuales}

La segregación residencial, entendida como una relación espacial, refiere a la separación o proximidad territorial entre personas o familias pertenecientes a un mismo grupo social (Sabatini, Cáceres \& Cerda, 2004). Su dinámica permite comprender como las desigualdades sociales se plasman en el espacio urbano me-

\footnotetext{
5 Pbro. Gustavo Carrara, responsable de la Vicaría de Villas de la Arquidiócesis, en su presentación en la reunión "no más chicos descartables, construyendo esperanza", remarcó que en el proyecto de las tres "C": colegio, capilla y club, fue de gran relevancia la visión y el impulso del cardenal Bergoglio.

6 La investigación fue apoyada con un subsidio del Intercambio Cultural Alemania-América Latina (ICALA) y otro de la Universidad Católica Argentina

7 CABA se encuentra dividida en 15 comunas. Las dos escuelas estudiadas están emplazadas en las villas ubicadas en las comunas 7 y 8 . Son comunas ubicadas en el sur de la ciudad, con indicadores sociodemográficos más deficitarios que los del resto de la ciudad.
} 
diante la relegación de los grupos más pobres a barrios con menor cobertura en bienes y servicios. Habitar en territorios segregados con alta concentración de pobreza pareciera producir un cúmulo de desventajas, como varios estudios vienen demostrando (Kaztman \& Queiroz Ribeiro, 2008; Groisman \& Suárez 2009; Veleda, 2005), A las carencias en los activos personales y familiares, se suma la penalidad que comporta habitar en territorios que en general tienen serios problemas de accesibilidad y conectividad, lo que dificulta la obtención de servicios y el acceso a las fuentes de trabajo y educación.

Las consecuencias de la segregación residencial, conocidas en la literatura como "efectos vecindario", son particularmente negativas para los sectores más pobres al converger con otra dinámica que expresa desigualdad social: la segmentación educativa. Este concepto remite "tanto a la presencia de circuitos escolares de distinta calidad, como a la homogeneidad en la composición socioeconómica del público al que atienden" (Krüger, 2014, p.1). ${ }^{8}$

El Sistema Educativo argentino ha sido crecientemente inclusivo; proceso que se vio reflejado en constantes aumentos en la tasa de escolarización formal. La masividad del nivel primario se alcanzó mucho antes que en la mayoría de los países de la región. Desde mediados del siglo XX, y particularmente en las décadas de 1980 y 1990 se fue incrementando la matrícula del nivel medio; la obligatoriedad de este nivel se estableció en $2006 . .^{9}$ Si bien la creciente incorporación de sectores históricamente marginados constituye un logro, numerosos estudios evidencian una desigual distribución de oportunidades en términos de equidad educativa (López, 2005).

La desigualdad en el origen social de las personas, en sus condiciones de vida, en sus trayectorias o en sus pertenencias culturales pone en cuestión la pertinencia de una oferta educativa igual para todos. El principio de igualdad que primó durante la conformación del sistema educativo debería ser reemplazado por otro, presentado por algunos autores como superador, el de equidad. Este consiste, según Rivas (2010) en la posibilidad de brindar respuesta específica a problemas específicos, mientras que para López, la equidad educativa, consiste en

partir de un profundo conocimiento de las situaciones en que se dan las prácticas de enseñanza y aprendizaje, y de las características sociales y culturales de las comunidades... [En este sentido] lo local adquiere especial centralidad frente al desafío de la equidad. (López, 2005, p. 65)

\footnotetext{
8 La segmentación educativa comenzó a ser objeto de estudio en Argentina a partir del aporte pionero de Cecilia Braslavsky (1985), quien constató la existencia de continuidades entre la fragmentación social y los procesos de diferenciación horizontal de las instituciones educativas. El concepto, desde su perspectiva teórico-político de tinte humanista, permite comprender la relación entre el nivel macro y el nivel micro educativo, el sistema y las instituciones, los procesos sociales y los procesos individuales.

${ }^{9}$ En el 2006 se promulgó en Argentina una nueva Ley de Educación que estableció la obligatoriedad de nivel medio; se promovieron políticas de inclusión social destinadas a garantizar el acceso real y la permanencia en la educación formal de jóvenes en situación educativa de riesgo (Llosa, Sirvent Toubes \& Santos, 2001, p. 8).
} 
Las consecuencias negativas de una creciente inclusión educativa, sin equidad, en el nivel medio, en paralelo a la consolidación de la segmentación del sistema educativo -como fue el caso en Argentina-, son variadas y van en diversas direcciones. Una de ellas es que se debilita el papel cohesionador e integrador social de la escuela, en el que los estudiantes aprenden a convivir con personas de distinta condición económica, social y cultural (Gasparini, Jaume \& Vázquez, 2011; Kaztman \& Queiroz Ribeiro, 2008; Kessler, 2002; Rivas 2010, entre otros). Desde lo institucional varios estudios evidencian cómo las propias escuelas a través de sus prácticas cotidianas contribuyen a la profundización de la segregación/segmentación (Veleda, 2005) lo que obliga a crear nuevos formatos (Nóbile, 2014, 2016; Montes \& Ziegler, 2010; Tiramonti, 2011; Briscioli, 2015). A nivel pedagógico, otra consecuencia relevante la constituye la falta de un currículum pertinente cuya función no sea la transmisión y reproducción cultural sino la resistencia y producción cultural (De Alba, 1998) que tenga en cuenta saberes previos, aprendizajes situados donde el contexto cultural y la participación en las actividades tienen profundas repercusiones en el aprender y conocer (Lenon de Villar, 2003; Wenger, 2001; Rogoff, 2012). Consecuencia directa de ello es la falta de calidad de los contenidos y de los procesos cognitivos (Rosetti, 2014; Facciola \& Aguilar Rivera, 2015). Dentro de este nivel cabe asimismo mencionar la incidencia de lo que la literatura identifica como "efecto entre pares", es decir la composición de los alumnos que comparten un aula-escuela ya que afecta los resultados educacionales obtenidos (Valenzuela, 2008; Bellei, 2013).10 Los procesos cognitivos están íntimamente relacionados con el "aprendizaje situado", entendido como la actividad en contexto. Cobra así un potencial significativo en la inserción sociolaboral de los jóvenes provenientes de poblaciones vulnerables (Sagástegui, 2004; Niemeyer, 2006). La adquisición de competencias como resultado del aprendizaje, desde esta perspectiva, no se consolida en el saber sino en la participación activa de los alumnos en las actividades colectivas.

La interrelación entre segregación residencial y segmentación educativa aumenta la vulnerabilidad social de los jóvenes que las transitan. Estas poblaciones vulnerables son "grupos de personas que, a consecuencia de las condiciones del medio en que viven, están en una situación de mayor susceptibilidad al daño" (Feito, 2007, p. 8). El término vulnerabilidad concebido en su dimensión social conduce a hablar de las capacidades y el reconocimiento como elementos clave del vínculo entre los seres humanos que es fundamento de la obligación moral.

En síntesis, los logros educativos son un producto combinado de factores de socialización de sus hogares, de sus vecindarios y de los establecimientos de enseñanza a los que asisten (Kaztman \& Queiroz Ribeiro, 2008). Cada uno de estos ámbitos puede representarse, por un lado, como un conjunto de activos en capital cultural, social, económico y simbólico (Bourdieu, 1987), así como de capital

\footnotetext{
10 Los compañeros de aula-escuela ejercen múltiples tipos de efectos sobre la educación de un alumno particular, los cuales se pueden clasificar en "efectos sobre el ambiente para el aprendizaje (contexto) y efectos sobre los procesos de enseñanza-aprendizaje; a su vez, es posible argumentar que dichos efectos pueden operar por canales diferentes, lo que permite clasificarlos en efectos directos sobre el alumno en cuestión y efectos indirectos o mediados por otros" (Bellei, 2013, p. 328).
} 
espiritual (Grace, 2007) y, por otro, como un conjunto de capacidades organizacionales que facilitan en mayor o menor medida la transformación de esos activos en recursos para la socialización de los jóvenes.

El argumento expuesto hasta aquí considera que la segregación socioeconómica y la segmentación educativa constituyen factores relevantes de la (in)equidad educativa, en tanto afectan diferencialmente las oportunidades, los logros de aprendizaje de los alumnos y el desarrollo de competencias laborales (Gallart, 2010). La creciente inclusión educativa en paralelo al incremento en la segmentación del sistema escolar tiende a redundar en que la inequidad social se exprese al interior del sistema educativo, reproduciendo así la desigualdad de origen de los alumnos. Escuelas del nivel medio ubicadas en espacios urbanos altamente segregados que reciben a alumnos de los sectores sociales más marginados, provenientes de circuitos educativos segmentados -como es el caso de las dos que forman parte de nuestro estudio-, enfrentan desafíos particulares para garantizar calidad educativa multidimensional y contextual (Arocho Rodriguez, 2010).

Desde este marco conceptual cobran sentido las preguntas teóricas que enmarcan nuestro trabajo: ¿cómo las escuelas en espacios marginales ayudan a articular el territorio de la periferia con el del resto de la ciudad?, ¿en qué medida favorecen al reconocimiento social de los alumnos?, ¿cómo es el involucramiento de los diversos actores sociales en la tarea educativa que se hace desde estas escuelas?, ¿cómo articulan inclusión, contención y reconocimiento social con calidad educativa?, ¿cuáles son los aprendizajes, los valores y los procesos de construcción de subjetividad en las escuelas que favorecen el encuentro entre los actores de las diversas culturas?, ¿cómo hacen para que los alumnos logren atesorar capital cultural, social y espiritual, y que estos capitales los ayuden a una adecuada inclusión social?

Las escuelas sobre las que se centra nuestro trabajo reciben a una población marginal o en situación educativa de riesgo, que ha transitado por circuitos de baja calidad del sistema educativo; es una población residente en espacios segregados de la ciudad hacia donde ellas también se han emplazado. Tienen el desafío de favorecer un aprendizaje que ofrezca la oportunidad de revertir el déficit acumulado por los alumnos. Las motivaciones, las visiones y las herramientas pedagógicas con que encaran la tarea cobran así relevancia, lo mismo que la inquietud sobre los logros que van obteniendo.

\section{Método, muestra e instrumentos}

Nuestro estudio se enmarcó en la tradición de estudio de caso. ${ }^{11}$ Elegimos dos establecimientos educativos de gestión privada ubicados en territorios de alta

${ }^{11}$ El estudio de caso es un examen completo o intenso de una faceta, una cuestión o acontecimientos que tienen lugar en un marco geográfico a lo largo del tiempo. Comporta una investigación procesual sistemática y profunda de un caso en concreto. Su abordaje se caracteriza por el uso de una variedad de fuentes primarias y secundarias de datos (ver Stake, 2005). 
marginalidad del sur de la ciudad: $M M P$ en la villa del Bajo Flores, VI en Ramón Carrillo, ambos sostenidos por sacerdotes diocesanos de la Vicaría de Villas de la Arquidiócesis de Buenos Aires. Estas dos escuelas junto a otras tres conforman las escuelas parroquiales presentes en villas de CABA. ${ }^{12}$ Ambas escuelas están en la zona sur de la ciudad. MMP se ubica dentro de la Comuna 7 , en una de las tres villas más grandes de CABA, conocida también como la 1-11-14. La escuela VI está dentro de la Comuna 8 que es aquella que concentra el mayor porcentaje de villas de la ciudad.

El procedimiento del estudio en las dos escuelas seleccionadas consistió en tomar contacto con los sacerdotes involucrados, directivos y responsables pedagógicos de las escuelas. Se entabló un fluido diálogo entre nuestro equipo y ellos, de manera de diseñar un trabajo y reflexión relevante. Fruto de este intercambio se terminaron de confeccionar dos cuestionarios diseñados por nuestro equipo (uno sociodemográfico y otro axiológico); estos fueron revisados y ampliados en términos tanto de los datos a relevar como de la claridad en la expresión lingüística. El cuestionario sociodemográfico incluyó las siguientes dimensiones: 1. Datos básicos de los alumnos (edad, sexo, lugar de nacimiento y datos de migración); 2. Datos de sus padres (nivel educativo, ocupación, ingresos y migración); 3. Composición del hogar; 4. Trayectoria educativa (repitencias, abandonos, cambios de escuelas); 5. Motivos para la elección de la escuela. El cuestionario axiológico se basó en el de valores interpersonales de L. Gordon (1978, versión adaptada y validada a la población argentina por Moreno y Rodríguez (Moreno, 2000). El objetivo fue apreciar los valores que los sujetos poseen o adoptan y que se pueden considerar como un aspecto de la configuración de su personalidad. Las dimensiones relevadas fueron: 1 . Independencia; 2 . Benevolencia; 3. Estímulo; 4. Conformidad Social; 5. Liderazgo; 6. Reconocimiento Social.

Posteriormente se presentó el proyecto de investigación a los alumnos y con su consentimiento informado, se procedió a la toma del cuestionario. Este fue auto-aplicado bajo nuestra supervisión. Los alumnos respondientes fueron 107, lo que representa a más del $90 \%$ del alumnado matriculado en estas escuelas en el 2016; quedaron fuera solo aquellos alumnos que no asistieron a la escuela el día del relevamiento.

Asimismo en ambas escuelas hicimos entrevistas en profundidad al equipo directivo: director, representante legal, asesora pedagógica, y preceptores en tanto informantes clave. Se ahondó en sus visiones, motivaciones y en el proyecto pedagógico, sus desafíos y balances.

\footnotetext{
12 Las otras tres escuelas parroquiales en villas de CABA son: el Instituto Parroquial Nuestra Señora del Carmen en Ciudad Oculta; el Instituto Parroquial Virgen de los Milagros de Caacupé en la Villa 21 perteneciente a la parroquia Virgen de los Milagros de Caacupé; y un jardín de infantes en la Villa Inta (Villa 19, Manzana 3) Centro comunitario San Cayetano. Los 5 establecimientos educativos parroquiales en villas son parte de los 64 establecimientos que pertenecen a la Vicaria Episcopal de Educación, es decir, que la entidad propietaria es el Arzobispado de Buenos Aires. De estas escuelas, 42 son parroquiales.
} 
Los datos provenientes de los cuestionarios fueron procesados y analizados estadísticamente con el software SPSS. Cabe señalar que con los datos del cuestionario sociodemográfico, donde era posible hacer comparaciones, estas se hicieron con datos provenientes de la Encuesta Anual de Hogares de la Dirección de Estadísticas de la Ciudad de Buenos Aires, onda 2015, y con datos de la Unidad de Evaluación Integral de la Calidad y Equidad Educativa. Ministerio de Educación del GCBA. Relevamiento Anual 2015.

\section{Resultados}

\section{Perfil sociodemográfico y trayectorias educativas de los alumnos de las escuelas MMP y VI}

El nivel educativo de los padres de los alumnos evidencia el perfil socioeconómico de sus familias. La fuerte concentración en los niveles de instrucción más bajos marca una brecha con los adultos de las comunas a las que pertenecen las escuelas, y particularmente con el total de los adultos de CABA. Su origen de estratos bajos se combina con la diversidad cultural de sus procedencias, en la que se destaca la matriz latinoamericana (especialmente Paraguay, Bolivia y Perú) -proporción que duplica a la de migrantes latinoamericanos de las comunas 7 y 8 , y casi quintuplica a la del total de CABA-. Los alumnos de las escuelas de este estudio tienden a vivir en hogares numerosos, de casi 6 miembros en promedio, valor que duplica al de los hogares de CABA. Cabe destacar que aproximadamente un tercio de los alumnos desconocen datos respecto a sus papás; lo que podría indicar ausencia de esta figura en sus trayectorias de vida. A su vez solo la mitad comparte el hogar con su padre -proporción solo levemente más baja a la de los adolescentes de las comunas 7 y 8 , y a la del total de CABA-; un 10 por ciento comparte el hogar con el padrastro y $37,1 \%$ no vive ni con su padre ni con un padrastro en su hogar. Se destaca asimismo el alto porcentaje que vive en hogares familiares extendidos $(37,1 \%)$ con presencia de tíos, abuelos u otros parientes. La vulnerabilidad laboral de los papás de los alumnos en estudio se evidencia en la mayor dificultad que tienen para insertarse laboralmente $-17,7 \%$ no está en el mercado de trabajo- en relación a adultos varones del resto de CABA, y en la precariedad de los trabajos en los que se desempeñan (ver Tabla 1). Puede resumirse de lo analizado hasta aquí que las escuelas de nuestro estudio tienden a captar a adolescentes provenientes de hogares con alta vulnerabilidad social y por tanto en riesgo de no lograr apoyar la escolaridad media de sus miembros adolescentes.

La edad promedio de los alumnos encuestados es de 15 años. Valor alto si se considera que un $80 \%$ cursa 1 . ero o 2 . do año. La sobreedad que este dato evidencia es uno de los indicadores de la situación de vulnerabilidad educativa en la que se encuentran los alumnos. Dos tercios de los matriculados son varones..$^{13}$ Asisten a ambas escuelas adolescentes que en su mayoría nacieron en Argentina (72,6\%). Poco más de un cuarto nació en Paraguay, Bolivia o Perú, países desde los cuales,

\footnotetext{
13 La mayor presencia de varones puede estar asociada al proyecto educativo de estos nuevos establecimientos vinculados a la recuperación de jóvenes con problemas de adicción.
} 
en la mitad de los casos, estos adolescentes llegaron recientemente a la Argentina. Sumando a este dato el de aquellos adolescentes que menos de cinco años atrás vivían fuera del barrio actual, se conforma un tercio de los alumnos que llegaron al barrio actual recientemente. Cerca de un quinto de los alumnos trabaja paralelamente a asistir a la escuela; proporción muy superior a la del resto de los alumnos de CABA. Sus inserciones laborales son precarias; muchos ayudan a sus padres en trabajos de construcción, limpieza o en sus locales de venta en los barrios. Un grupo pequeño de los alumnos tiene hijos (4,7\%) (Ver Tabla 2).

Tabla 1. Datos básicos de los padres de los alumnos de MMP y VI, de varones entre 30-55 años del total de CABA y de las Comunas 7 y 8

\begin{tabular}{|c|c|c|c|c|}
\hline & \multicolumn{2}{|c|}{ MMP y VI } & \multirow{2}{*}{$\begin{array}{l}\text { Comunas } \\
7 \text { y } 8(*) \\
\text { Padre } \\
\end{array}$} & \multirow{2}{*}{$\begin{array}{c}\text { Total CABA } \\
(*) \\
\text { Padre } \\
\end{array}$} \\
\hline & Madre & Padre & & \\
\hline \multicolumn{5}{|l|}{ Má } \\
\hline \multirow{2}{*}{$\begin{array}{l}\text { Sin instrucción y primario incomp. } \\
\text { Primario completo }\end{array}$} & $28,1 \%$ & $26,9 \%$ & $3,4 \%$ & $1,6 \%$ \\
\hline & $13,5 \%$ & $15,9 \%$ & $11,6 \%$ & $7,3 \%$ \\
\hline \multirow{3}{*}{$\begin{array}{l}\text { Secundario incompleto } \\
\text { Secundario completo y más } \\
\text { Total }\end{array}$} & $33,7 \%$ & $28,6 \%$ & $18,4 \%$ & $11,3 \%$ \\
\hline & $24,8 \%$ & $28,5 \%$ & $66,6 \%$ & $79,7 \%$ \\
\hline & $\begin{array}{c}100 \% \\
(89)\end{array}$ & $\begin{array}{c}100 \% \\
(63)\end{array}$ & $\begin{array}{c}100 \% \\
(68646)\end{array}$ & $\begin{array}{c}100 \% \\
(502061)\end{array}$ \\
\hline Desconoce el dato o no contesta & $16,6 \%$ & $41,1 \%$ & & \\
\hline \multicolumn{5}{|l|}{ Lugar de nacimiento } \\
\hline \multirow{2}{*}{$\begin{array}{l}\text { Argentina } \\
\text { Paraquay }\end{array}$} & $37,8 \%$ & $43,2 \%$ & $76,4 \%$ & $84,2 \%$ \\
\hline & $33,7 \%$ & $32,4 \%$ & \multirow{3}{*}{$22,6 \%$} & \multirow{3}{*}{$13,0 \%$} \\
\hline Bolivia & $22,4 \%$ & $16,2 \%$ & & \\
\hline \multirow{3}{*}{$\begin{array}{l}\text { Otros países limítrofes y Perú } \\
\text { Otro país } \\
\text { Total }\end{array}$} & $6,2 \%$ & $6,9 \%$ & & \\
\hline & $0 \%$ & $1,4 \%$ & $1,0 \%$ & $2,8 \%$ \\
\hline & $\begin{array}{c}100 \% \\
(98)\end{array}$ & $\begin{array}{c}100 \% \\
(74)\end{array}$ & $\begin{array}{c}100 \% \\
(68646)\end{array}$ & $\begin{array}{c}100 \% \\
(502061)\end{array}$ \\
\hline Se desconoce el dato & $8,4 \%$ & $31 \%$ & & \\
\hline \multicolumn{5}{|l|}{ Trabaja actualmente } \\
\hline $\begin{array}{l}\text { No } \\
\text { Sí }\end{array}$ & $55,2 \%$ & $82,3 \%$ & $92,2 \%$ & $94,6 \%$ \\
\hline Total & $\begin{array}{l}100 \% \\
(105)\end{array}$ & $\begin{array}{c}100 \% \\
(79)\end{array}$ & $\begin{array}{c}100 \% \\
(68646)\end{array}$ & $\begin{array}{c}100 \% \\
(502061)\end{array}$ \\
\hline
\end{tabular}

(*) Para aproximarnos a una población comparable con la de los padres de los alumnos de las escuelas de nuestro estudio, tomamos a adultos entre 30 y 55 años.

Fuente: Elaboración propia en base a los datos obtenidos de las encuestas realizadas los días 6 y 22 de septiembre de 2016 a 107 alumnos de las escuelas MMP y VI y la Encuesta Anual de Hogares 2015 (base usuarios ampliada) de la Dirección General de Estadísticas y Censos de CABA. 
Tabla 2. Datos básicos de los alumnos de MMP y VI, de los alumnos de las Comunas 7 y 8, y del total de alumnos de las escuelas de CABA

\begin{tabular}{|c|c|c|c|}
\hline & MMP y VI & $\begin{array}{l}\text { Comunas } \\
7 \text { y } 8(*)\end{array}$ & $\begin{array}{c}\text { Total CABA } \\
(*) \\
\end{array}$ \\
\hline \multicolumn{4}{|l|}{ Sexo } \\
\hline \multirow{3}{*}{$\begin{array}{l}\text { Mujer } \\
\text { Varón } \\
\text { Total }\end{array}$} & $36,4 \%$ & $54,6 \%$ & $52,3 \%$ \\
\hline & $63,6 \%$ & $45,4 \%$ & $47,7 \%$ \\
\hline & $100 \%(107)$ & $100 \%(21258)$ & $100 \%(109781)$ \\
\hline \multicolumn{4}{|l|}{ Edad } \\
\hline \multirow{2}{*}{$\begin{array}{l}\text { Promedio } \\
\text { Rango de edad }\end{array}$} & 15 & 14,8 & 14,7 \\
\hline & $13-18$ & $12-19$ & $12-19$ \\
\hline \multicolumn{4}{|l|}{ Lugar de nacimiento } \\
\hline \multirow{2}{*}{$\begin{array}{l}\text { Argentina } \\
\text { Paraguay } \\
\text { Bolivia }\end{array}$} & $18,9 \%$ & \multirow{3}{*}{$16,9 \%$} & \multirow{3}{*}{$8,9 \%$} \\
\hline & $7,5 \%$ & & \\
\hline \multirow{3}{*}{$\begin{array}{l}\text { Otros países limítrofes y Perú } \\
\text { Otro país } \\
\text { Total }\end{array}$} & $0,9 \%$ & & \\
\hline & $0 \%$ & $0 \%$ & $2,0 \%$ \\
\hline & $100 \%(106)$ & $100 \%(21258)$ & $100 \%(109781)$ \\
\hline \multirow{5}{*}{$\begin{array}{l}\text { Donde vivía hace } 5 \text { años } \\
\text { En el mismo barrio } \\
\text { En otro lugar de Argentina } \\
\text { En Paraguay, Bolivia o Perú } \\
\text { Otro país no limítrofe ni Perú } \\
\text { Total }\end{array}$} & $67,6 \%$ & & \\
\hline & $18,1 \%$ & & \\
\hline & $13,4 \%$ & \multirow[b]{2}{*}{$6,3 \%$} & \multirow[b]{2}{*}{$2,6 \%$} \\
\hline & $1,0 \%$ & & \\
\hline & $100 \%$ & & \\
\hline Porcentaje que trabaja & $19,8 \%$ & $2,9 \%$ & $2,1 \%$ \\
\hline \multirow{2}{*}{$\begin{array}{l}\text { Tamaño de sus hogares } \\
\text { Promedio de personas por hogar } \\
\text { Rango }\end{array}$} & 5,9 & $3,5(* *)$ & $3,1(* *)$ \\
\hline & 1 a 15 & & \\
\hline \multicolumn{4}{|l|}{ Presencia del padre } \\
\hline \multirow{2}{*}{$\begin{array}{l}\text { Vive con el padre } \\
\text { Vive con padrastro } \\
\text { No vive ni con padre ni padrastro }\end{array}$} & $10,5 \%$ & & \\
\hline & $37,1 \%$ & & \\
\hline \multirow{5}{*}{$\begin{array}{l}\text { Composición de sus hogares } \\
\text { Nuclear familiar } \\
\text { Nuclear ensamblado } \\
\text { Nuclear incompleto } \\
\text { Familiar extendido } \\
\text { Con componentes no familiares }\end{array}$} & $33,3 \%$ & & \\
\hline & $10,5 \%$ & & \\
\hline & $18,1 \%$ & & \\
\hline & $37,1 \%$ & & \\
\hline & $1,0 \%$ & & \\
\hline
\end{tabular}

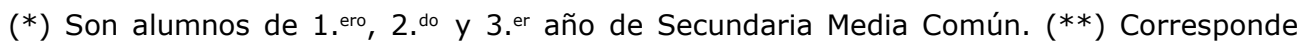
al tamaño de hogares familiares. Se descartan asimismo los hogares unipersonales.

Fuente: Elaboración propia en base a los datos obtenidos de las encuestas realizadas los días 6 y 22 de septiembre de 2016 a 107 alumnos de las escuelas MMP y VI, y la Encuesta Anual de Hogares 2015 (base usuarios ampliada) de la Dirección General de Estadísticas y Censos de CABA. 
Las trayectorias escolares de los alumnos de ambas escuelas indican tendencia a la repitencia y al cambio de escuela durante la primaria para alrededor de la mitad de ellos. En cuanto a la trayectoria en el nivel secundario, más de la mitad de los alumnos cursaron un año en otra escuela antes del ingreso a la actual. Entre los motivos predominantes se encuentra la repetición y el abandono escolar, ambos indicadores de riesgo educativo. De hecho, un 43,8\% repitió al menos un año del nivel medio; porcentaje muy superior al de los alumnos de este nivel en el resto de CABA. Se observa asimismo tendencia a desgranamiento de alumnos a medida que avanzan los años de escolarización secundaria ${ }^{14}$ y la segregación escolar (ver Tabla 3).

El alumnado de estas escuelas tiende en su mayoría a vivir en las inmediaciones de los establecimientos educativos. Poco más de un tercio participa en otras actividades recreativas y de animación que ofrecen las parroquias, aun si varios de ellos no se identifican con la religión católica ${ }^{15}$ (ver Tabla 3).

El cuestionario incluyó una pregunta abierta en la que se pedía a los jóvenes que indicaran por qué habían elegido la escuela. Las respuestas fueron variadas. Estas tienden a ponderar la cercanía $(35,5 \%)$; la propia elección $(31,8 \%)$ destacando el trato y la contención; expresadas en frases como "(lo elegí) porque es un buen colegio donde todos los profes, directores y preceptores te brindan un cariño, amor, comprensión, etc."; (lo elegí) "porque me siento cómodo con la maestra y no me siento excluido entre mis compañeros". Finalmente, un 30,8\% destaca que no fue una elección suya sino de algún pariente, que en general es la madre. Las respuestas de los alumnos pueden ser leídas de diferentes formas no excluyentes entre sí. Por un lado, podrían indicar que se trata de elecciones que ayudan a facilitar el tránsito escolar dada la cercanía de los establecimientos a sus lugares de residencia. Pueden, asimismo, indicar que son elecciones motivadas en la percepción de que estas escuelas contienen a quien está por abandonar la escuela. En este caso puede argumentarse que se trata de escuelas en espacios segregados que constituyen el último eslabón en la cadena de exclusión de jóvenes que el sistema educativo no logra retener (ver Tabla 3).

\section{Perfil de valores interpersonales para la convivencia y actitud hacia el apren- dizaje escolar}

La educación en valores en la sociedad actual, y en los contextos de alta vulnerabilidad, sigue siendo uno de los retos más importantes del Sistema Educativo.

\footnotetext{
14 Diversos estudios en Argentina muestran cómo el riego educativo aumenta en los adolescentes de los sectores más marginados. La repitencia, la sobreedad, el abandono y el desgranamiento, son indicadores de este riesgo. Entre otro, cabe mencionar el informe Programa de las Naciones Unidas para el Desarrollo [PNUD], 2010.

15 Solo dos tercios de los jóvenes encuestados expresan identificarse con alguna religión (69.1\%), en primer lugar con el catolicismo $(61.7 \%)$. Este porcentaje de alumnado católico es un poco menor al de los jóvenes residentes en villas de CABA (ver Suárez, 2015). Es destacable este dato ya que evidencia el carácter inclusivo de la oferta de las escuelas católicas en estudio.
} 
La convivencia en los contextos escolares está presidida por los valores que cada individuo le concede a su relación con los demás y al beneficio que quieran obtener de esa relación de allí que se manifiesta en una diversidad de conductas que van desde las más adaptadas hasta las menos deseables, desadaptativas 0 antisociales, que pueden favorecer o no el aprendizaje, la escolarización y la inclusión social.

Tabla 3. Datos de las trayectorias educativas de los alumnos

\begin{tabular}{|c|c|c|c|}
\hline & MMP y VI & $\begin{array}{l}\text { Comunas } \\
7 \text { y } 8(*)\end{array}$ & $\begin{array}{c}\text { Total CABA } \\
(*)\end{array}$ \\
\hline \multicolumn{4}{|l|}{$\begin{array}{l}\text { Cantidad de escuelas en las } \\
\text { que cursó la primaria }\end{array}$} \\
\hline $\begin{array}{l}\text { Una } \\
\text { Dos o más (con rango 1-5) }\end{array}$ & $\begin{array}{l}52 \% \\
48 \%\end{array}$ & & \\
\hline \multirow{2}{*}{$\begin{array}{l}\text { Repitió algún grado de prim. } \\
\text { No } \\
\text { Sí }\end{array}$} & $70 \%$ & & \\
\hline & $30 \%$ & & \\
\hline \multicolumn{4}{|l|}{$\begin{array}{l}\text { Fue a alguna otra escuela } \\
\text { secundaria antes de ingresar } \\
\text { a la actual }\end{array}$} \\
\hline $\begin{array}{l}\text { No } \\
\text { Sí }\end{array}$ & $53,9 \%$ & & \\
\hline \multirow{2}{*}{$\begin{array}{l}\text { Repitió algún año de secund. } \\
\text { No } \\
\text { Sí }\end{array}$} & $56,2 \%$ & $87 \%$ & $89,1 \%$ \\
\hline & $43,8 \%$ & $13 \%$ & $11,9 \%$ \\
\hline \multicolumn{4}{|l|}{$\begin{array}{l}\text { A qué distancia queda el } \\
\text { colegio actual de su casa }\end{array}$} \\
\hline \multirow{3}{*}{$\begin{array}{l}\text { Hasta } 400 \text { metros } \\
\text { Entre } 400 \text { y } 800 \text { metros } \\
\text { Entre } 800 \text { y } 1500 \text { metros } \\
\text { A más de } 15 \text { cuadras }\end{array}$} & $\frac{41,2 \%}{22,5 \%}$ & & \\
\hline & $\begin{array}{l}22,5 \% \\
16,7 \%\end{array}$ & & \\
\hline & $19,6 \%$ & & \\
\hline \multirow{4}{*}{$\begin{array}{l}\text { Motivos del alumno par } \\
\text { elección de la escuela } \\
\text { La eligió } \\
\text { Cercanía a su casa } \\
\text { No fue una elección suya } \\
\text { No sabe }\end{array}$} & $31,8 \%$ & & \\
\hline & $35,5 \%$ & & \\
\hline & $30,8 \%$ & & \\
\hline & $1,9 \%$ & & \\
\hline
\end{tabular}

(*) Datos correspondientes a alumnos de 2. do año.

Fuente: Elaboración propia en base a los datos obtenidos de las encuestas realizadas los días 6 y 22 de septiembre de 2016 a 107 alumnos de las escuelas MMP y VI, y la Encuesta Anual de Hogares 2015 (base usuarios ampliada) de la Dirección General de Estadísticas y Censos de CABA, y los datos provenientes del Relevamiento Anual 2015 de la Unidad de Evaluación Integral de la Calidad y Equidad Educativa. Ministerio de Educación del GCBA. 
Entendemos que los valores actúan como marco de referencia común. Describir el "perfil de valores interpersonales" es caracterizar de manera preliminar el sistema de valores que mueve a los sujetos a actuar y se encuentra asociado a un conjunto de objetivos educativos con o sin mención de ello. Los valores interpersonales se definen como "convencimientos sostenidos en el tiempo" que una persona posee respecto de un determinado modo de conducta preferente. Esta idea sobre el papel de los valores interpersonales en la conducta permite afirmar que la carencia de estos y de conductas prosociales se relaciona con conductas desadaptativas.

Asimismo, los valores interpersonales están asociados a las actitudes, entendidas como esta tendencia favorable o desfavorable hacia un objeto de representación que se expresa como un componente motivacional afectivo. Por ello consideramos importante y necesario indagar acerca del tema para poner en marcha intervenciones psico-socio-pedagógicas que sirvan para fomentar valores prosociales en los sujetos escolarizados con el fin de incrementar los valores democráticos y la inclusión social desde la diversidad.

Para la obtención del perfil de valores interpersonales se utilizó la versión abreviada del Cuestionario de Valores Interpersonales de Gordon adaptada y validada por Moreno (Moreno, 2000), que fue aplicado a la muestra anteriormente descrita, correspondiente a los alumnos de ambas escuelas.

En orden a considerar si el instrumento mide lo que pretende en la muestra seleccionada obtuvimos el coeficiente de alfa de Cronbach (1951). El mismo permite considerar que cuanto más cerca se encuentre el valor de alfa a 1, mayor es la consistencia interna de los ítems analizados. Los coeficientes obtenidos para el cuestionario de valores interpersonales fue un nivel muy respetable dado que los valores obtenidos fueron en su mayoría por encima de .70 para la muestra, observándose un valor levemente descendido en la dimensión Independencia (.60) (ver Tabla 4).

Tabla 4. Clasificación de las dimensiones por peso del Coeficiente de Alpha de Cronbach

\begin{tabular}{|l|c|}
\hline Dimensión & Coeficiente de Alpha \\
\hline Conformidad Social & 0.856 \\
\hline Reconocimiento social & 0.817 \\
\hline Benevolencia & 0.792 \\
\hline Estímulo o Soporte & 0.772 \\
\hline Liderazgo & 0.728 \\
\hline Independencia & 0.604 \\
\hline
\end{tabular}

Fuente: Elaboración propia en base a los 107 alumnos encuestados en las escuelas MMP y VI. Relevamientos efectuados los días 6 y 22 de septiembre 2016. 
También realizamos el análisis de medias -o promedios- a nivel intragrupo, con el objeto de conocer el perfil de valores interpersonales, entre los que se encuentran en los niveles más altos de valoración las dimensiones de Independencia (M:21,80) y Benevolencia (M:21,48) Seguidos, en orden descendente, por la dimensión de Estímulo o Soporte (M:20,23) y Conformidad Social (M:19.61) cerrando la lista, y con bajos niveles de valoración, la dimensión de Liderazgo (M:16.15) y Reconocimiento Social (M:14.34). (Ver Tabla 5).

Esta aproximación nos permite realizar una primera inferencia acerca de las dimensiones que se encuentran en los extremos del perfil. La jerarquización de la Independencia -entendida como tener el derecho a hacer lo que se quiera-, como contracara del reconocimiento social -entendido como ser bien visto y considerado por los otros, así como también conseguir el reconocimiento de los demás-, pone en evidencia la necesidad de intervenciones educativas, psicológicas y sociales que fortalezcan el desarrollo de capacidades que posibiliten el reconocimiento social como fruto de procesos productivos que afiancen la imagen personal y orienten la independencia.

Todos los alumnos manifestaron una alta valoración hacia el aprendizaje escolar, expresada por casi el $70 \%$ como muy importante y un 30\% como importante a partir de lo cual puede inferirse una tendencia actitudinal favorable hacia el aprendizaje escolar, esto se constituye en un capital simbólico de relevancia.

Asimismo, se pidió a los alumnos de la muestra que justificaran su respuesta acerca de por qué era importante o muy importante aprender. Se obtuvieron los siguientes resultados: en primer lugar, para los alumnos entrevistados, el aprendizaje está asociado a la "proyección a futuro" (51.1\%), implica la posibilidad de pensar en tener un trabajo, estudiar, progresar como se ilustra a continuación: ${ }^{16}$ "es importante para continuar estudiando"; "es importante para hacer carrera", "es

Tabla 5. Clasificación de las dimensiones según peso de la media

\begin{tabular}{|l|c|c|}
\hline Dimensión & Media & Desviación estándar \\
\hline Independencia & 21,80 & 3,102 \\
\hline Benevolencia & 21,48 & 3,659 \\
\hline Estímulo & 20,23 & 4,068 \\
\hline Conformidad Social & 19,61 & 4,541 \\
\hline Liderazgo & 16,15 & 3,962 \\
\hline Reconocimiento Social & 14,34 & 4,530 \\
\hline
\end{tabular}

Fuente: Elaboración propia en base a los 107 alumnos encuestados en las escuelas MMP y VI. Relevamientos efectuados los días 6 y 22 de septiembre 2016.

16 Ilustraciones obtenidas de las preguntas abiertas del cuestionario de valores interpersonales. 
muy importante para tener una profesión linda" así como también se proyecta "tener un trabajo", "para mantener a la familia y no pasar necesidades", "ayudar económicamente a mi familia", "tratar de salir del mundo donde vivo". En segundo lugar, el significado asociado al aprendizaje más importante fue "ser alguien" (23.7\%) acompañado por afirmaciones tales como: "Aprendo porque quiero ser alguien", "Para que algún día pueda ser algo importante". Y el tercer significado relevante del aprendizaje está asociado con "entender, conocer, saber" (16.5\%) habilitando la dimensión "cognitiva" del aprendizaje como se ilustra a continuación "Aprender es muy importante porque me ayuda a entender", "a conocer muchas cosas", "te abre la mente", "es muy importante porque no solo aprendés a leer y a escribir sino también a hablar". Lo expresado remite al potencial integrador que tiene el aprendizaje para los sujetos y su posible concreción a través del espacio escolar.

Con el fin de lograr un mayor entendimiento acerca de los procesos valorativos en la muestra y las variables de contexto, se procedió a un análisis multivariado. Utilizamos para ello la distribución de Hotelling cuyo coeficiente, obtenido a través del uso de SPSS, nos permitió explorar, a partir del análisis de media, la relación estadísticamente significativa entre las variables (Ver Tabla 6).

En un primer nivel de análisis identificamos las variables independientes que influyen en los procesos valorativos: Sexo-Estímulo, Tramo etario-Conformidad Social; Nivel Educativo de la madre-Reconocimiento social y Liderazgo; Nivel Educativo del padre-Liderazgo; Situación Laboral del padre-Conformidad social, Benevolencia y Estímulo.

En un segundo nivel de análisis profundizamos en las diferencias de las medias para cada una de las relaciones mencionadas con anterioridad y llegamos a los siguientes resultados: 1 . Las alumnas mujeres consideran importante que se las trate con comprensión y amabilidad, y valoran recibir el apoyo por parte de los demás (Dimensión Estímulo) más que los varones. 2. Los alumnos y alumnas, menores de 15 años, consideran importante hacer lo que es socialmente correcto, siguiendo estrictamente las normas establecidas (Dimensión Conformidad Social). 3. Los alumnos y alumnas, cuyas madres nacieron en Argentina, valoran como importante el tener derecho a hacer lo que se quiere (Dimensión Independencia) por sobre los alumnos y alumnas que tienen madres que nacieron en otros países de la región. 4. Los alumnos y alumnas, cuyas madres y padres tienen un nivel medio de educación, es decir, han finalizado el secundario, valoran como importante el liderazgo entendido como la capacidad de tener a cargo personas con autoridad, poder estar a cargo de un puesto de trabajo o poder. Así como los que tienen una madre que finalizó el nivel secundario valoran ser bien vistos, ser considerados importantes o llamar favorablemente la atención de los demás. 5. Los alumnos y alumnas cuya situación laboral del padre es activa, independientemente del tipo del trabajo, valoran hacer cosas por los demás, ayudar y ser generosos (Benevolencia) así como ser bien tratados recibiendo comprensión y apoyo (Estímulo/Soporte) y hacer lo que es socialmente correcto (Conformidad Social).

En síntesis, los principales resultados obtenidos permiten afirmar que: 1. el perfil de valores interpersonales, de los alumnos que participaron en la investigación, 
manifiesta un nivel alto en Independencia y Benevolencia, un nivel medio en Estímulo y Conformidad Social y un bajo nivel en Liderazgo y Reconocimiento Social, éstos últimos requieren de estrategias de intervención específicas para su fortalecimiento desde la escuela; 2. la Actitud hacia el aprendizaje, manifiesta una franca tendencia valorativa siendo "muy importante" (69\%). Esta actitud está asociada a tres significados relevantes para los alumnos que se presentan en orden de importancia: a) la posibilidad de proyectar otro futuro posible; b) la construcción de la propia subjetividad en el espacio escolar y c) la posibilidad de desarrollar capacidades cognitivas tales como entender, conocer, saber; 3 ) en el análisis multivariado se encontraron diferencias significativas en todas las dimensiones de los valores interpersonales especialmente asociadas al sexo y edad de la muestra; así como también la incidencia del lugar de origen de la madre, el nivel educativo de los padres como la situación laboral del padre en los procesos de desarrollo de valores interpersonales.

Tabla 6. Análisis multivariado

\begin{tabular}{|c|c|c|c|c|}
\hline Variable independiente & $\begin{array}{c}\text { Variable } \\
\text { dependiente }\end{array}$ & $\begin{array}{c}\text { F. de } \\
\text { Hotteling }\end{array}$ & Sig. & Media \\
\hline \multirow[t]{2}{*}{ Lugar de nacimiento de la madre } & \multirow[t]{2}{*}{ Independencia } & \multirow[t]{2}{*}{5,392} & \multirow[t]{2}{*}{.022} & Nac. 22.74 \\
\hline & & & & Ext. 21.26 \\
\hline \multirow[t]{2}{*}{ Nivel Educativo de la madre } & \multirow[t]{2}{*}{ Reconoc. Social } & \multirow[t]{2}{*}{5,777} & \multirow[t]{2}{*}{.002} & Bajo 13.51 \\
\hline & & & & Medio 15.68 \\
\hline \multirow[t]{2}{*}{ Nivel Educativo de la madre } & \multirow[t]{2}{*}{ Liderazgo } & \multirow[t]{2}{*}{9,777} & \multirow[t]{2}{*}{.002} & Bajo 15.10 \\
\hline & & & & Medio 17.54 \\
\hline \multirow[t]{2}{*}{ Nivel Educativo del padre } & \multirow[t]{2}{*}{ Liderazgo } & \multirow[t]{2}{*}{3,388} & \multirow[t]{2}{*}{.038} & Bajo 16.34 \\
\hline & & & & Medio 17.89 \\
\hline \multirow[t]{2}{*}{ Sexo } & \multirow[t]{2}{*}{ Estímulo } & \multirow[t]{2}{*}{9,031} & \multirow[t]{2}{*}{.003} & Masc. 19.43 \\
\hline & & & & Fem. 21.91 \\
\hline \multirow[t]{2}{*}{ Situación Laboral del padre } & \multirow[t]{2}{*}{ Conform. Social } & \multirow[t]{2}{*}{6,549} & \multirow[t]{2}{*}{.013} & 20.25 \\
\hline & & & & No 16.71 \\
\hline \multirow[t]{2}{*}{ Situación Laboral del padre } & \multirow[t]{2}{*}{ Benevolencia } & \multirow[t]{2}{*}{4,583} & \multirow[t]{2}{*}{.036} & 21.83 \\
\hline & & & & No 19.36 \\
\hline \multirow[t]{2}{*}{ Situación Laboral del padre } & \multirow[t]{2}{*}{ Estímulo } & \multirow[t]{2}{*}{4,494} & \multirow[t]{2}{*}{.037} & 20.53 \\
\hline & & & & No 17.93 \\
\hline \multirow[t]{2}{*}{ Tramo etario } & \multirow[t]{2}{*}{ Conform. Social } & & & $-15 \quad 20.38$ \\
\hline & & & & +1618.20 \\
\hline
\end{tabular}

Fuente: Elaboración propia en base a los 107 alumnos encuestados en las escuelas MMP y VI. Relevamientos efectuados los días 6 y 22 de septiembre 2016 


\section{Proyectos educativos, visiones socio pedagógica, y motivaciones de los directivos de las dos escuelas}

Para el análisis cualitativo, siguiendo la propuesta de Alicia De Alba tuvimos en cuenta dos aspectos propios del currículum en tanto categorías previas. En primer lugar, consideramos los aspectos estructurales-formales, o sea los que hacen alusión a las disposiciones oficiales, programas de estudio y organización de la escuela; y, en segunda instancia, los aspectos procesuales-prácticos del currículum, los aspectos particulares tanto del barrio como de los sujetos.

\section{Aspectos estructurales formales del Proyecto Educativo Institucional (PEI) $y$ el Proyecto Curricular Institucional (PCI)}

La estructura, la organización y los contenidos de los PEI de cada una de las escuelas de nuestra investigación siguen los lineamientos oficiales y denotan la falta de variaciones sobre el formato institucional de escuela secundaria.

Lo mismo ocurre con el PCI. Se asume el currículum oficial de la Ciudad de Buenos Aires, esto significa que se tienen en cuenta, prioritariamente, los aspectos estructurales formales del sistema por sobre los específicos de la población y el contexto. No se observan adecuaciones significativas específicas tendientes a responder a las necesidades del sujeto y su contexto.

Esta falta de adecuación tanto del PEI como del PCI constituye una limitante para el éxito del proyecto de las escuelas. Esta falta de articulación entre aspectos específicos de la dimensión didáctica-áulica con las exigencias formales, colisionan, que como veremos a continuación, con la situación específica de los alumnos. Es contradictoria asimismo con la visión y motivaciones de los directivos de las escuelas.

\section{Aspectos específicos de la dimensión didáctica-áulica}

El modo de entender la dimensión didáctica-áulica de ambas escuelas responde con claridad a los requerimientos prácticos específicos de la población a la que asisten. Se inscriben en un compromiso social con la realidad inmediata a través de un marco de prácticas situadas. El rasgo relevante de estas prácticas es el carácter participativo de los alumnos en los procesos de construcción de la realidad "Tiene que ver con la búsqueda de la construcción del conocimiento; de lo cooperativo y lo comunitario inserto en la escuela, de los saberes previos; de construir entre todos el clima de aula, y los contenidos y las dinámica" (Asesora pedagógica de la escuela VI, septiembre de 2016). El modo de comprender al sujeto implícito en estas prácticas es holístico, en el que lo social, lo humano y lo trascendente están íntimamente relacionados (Facciola, 2017). Desde esta perspectiva específica se comprende a los sujetos como activos, sujetos de su historia que tienen un acervo cultural y toman decisiones sobre su futuro.

Las prácticas específicas, situadas en el contexto, están articuladas en redes solidarias con el club y la capilla con la expectativa de generar un círculo virtuoso entre educación, prevención y recreación. 
El recorrido de dos o tres años, desde que se fundaron las escuelas, permite que los propios directivos evalúen la acción educativa llevada adelante, tales como las dificultades en las normas de convivencia, entendidas como falta de disciplina -sobre todo en algunos casos como por ejemplo de violencia física y verbal dentro de la escuela-. Las problemáticas específicas de estos alumnos, tales como repitencia, sobreedad y el abandono escolar tanto esporádico como permanente, llevaron a pulir y orientar el desarrollo didáctico-áulico: "tuvimos que ir haciendo mezclas entre lo pedagógico, la búsqueda de orden de clima de estudio y de trabajo" (Asesora pedagógica de la escuela MMP, marzo de 2017); y agregó respecto a la normativa de convivencia "El año pasado hemos atravesado una prueba de fuego tremenda, había chicos muy difíciles... había que por un lado construir la normativa pero por otro lado no sabíamos qué enfoque queríamos construir".

Así el "desafío de congeniar lo ideal con lo real" (directivo de la escuela MMP, septiembre de 2016) en el desarrollo cotidiano de tener que enseñar impulsó a que estén tomando forma iniciativas didáctico-aúlicas centradas en los alumnos, en términos "de enseñar y adecuarnos al chico que tenemos enfrente... Acá hacemos esfuerzos enormes y eso es para nosotros -desde una perspectiva humanista-calidad educativa" (directivo de la escuela VI, septiembre de 2016).

En síntesis, se constata que en la dimensión didáctico-áulica se asume la problemática específica de la población a partir del enfoque de las pedagogías en situación (Barret, 1995, citado en Leitao, 2010) donde la actividad educativa tiene como centro al sujeto que aprende a partir de sus vivencias y contexto, considerando fundamentales las redes de contención para su promoción humana y la articulación con otras instancias para la promoción social.

Esto plantea una contradicción con los aspectos formales presentados en el PEI y los aspectos prácticos desarrollados en las aulas observándose una insuficiente articulación entre las dimensiones generales y específicas del currículum de las escuelas estudiadas.

\section{Motivaciones personales y visiones de los responsables de las escuelas}

Los directivos de las escuelas analizadas expresan una fuerte identificación con el proyecto educativo. Asimismo, la responsabilidad asumida está teñida de un doble compromiso: por un lado, construir una opción educativa significativa en un espacio de alta vulnerabilidad; por otro, la construcción de esta alternativa desde un espacio colectivo que le da forma a la propuesta.

De las visiones que los directivos expresaron, interesa destacar su forma de entender y proyectar la acción de la escuela en el barrio. Frases como "la escuela valoriza el barrio, no es lo mismo vivir en un barrio que tiene escuela secundaria que en un barrio que no la tiene" (director de la escuela MMP, abril de 2017) denotan una mirada que concibe la escuela no solo por lo que aporta a sus alumnos sino en su aporte en la construcción de la trama institucional del barrio; trama que va consolidando al barrio e integrándolo desde ahí al entorno urbano al que pertenece. Se trata de una mirada que "confía en que hay una potencialidad en estos barrios que va a ayudar a que salgan adelante" (director de la escuela MMP, abril de 2017). 
La construcción de una escuela secundaria en un barrio de alta marginalidad urbana, atendiendo a la literatura que enfatiza los "efectos de los vecindarios" en los logros educativos, sería altamente desaconsejable. La idea que la escuela valoriza al barrio cuestiona esta mirada.

\section{Conclusiones}

El estudio que efectuamos en dos escuelas secundarias de reciente creación muestra que efectivamente a estas concurren jóvenes vulnerables, en situación de riesgo social y educativo; jóvenes que muy posiblemente, de no mediar esta oferta inmersa en sus propios territorios de marginalidad, segregados residencialmente, estarían fuera de los circuitos educativos, conminados a tránsitos muy conflictivos. Provienen de hogares muy vulnerables; varios de ellos de reciente arraigo en el barrio y el país. El muy bajo nivel de instrucción de los padres de los alumnos es un indicador claro de vulnerabilidad que desafía a las escuelas de diversos sentidos. Por un lado, como institución debe contener y suplir un acompañamiento en la adquisición de conocimientos y en el proceso de aprendizaje que el hogar de los adolescentes está imposibilitado de ofrecer. Por otro lado, las pone ante el desafío de ir al encuentro de la carencia educativa de los adultos de los barrios, donde están emplazados los establecimientos, ofreciendo alternativas de educación no solo para niños y adolescentes sino también a adultos. ${ }^{17}$

Las escuelas estudiadas, situadas en contextos de alta marginalidad urbana, surgen como una propuesta político-educativa articulada al proyecto social de las parroquias insertas en los barrios. Su propuesta consiste en una manera de contribuir a la valorización de los barrios consolidando una institucionalidad -en articulación con otras instituciones que surgieron por iniciativa de la iglesia católica: el club y la capilla- que reconozca, confíe y acompañe a los jóvenes con el fin de dar respuesta a la inequidad social y educativa, y evitar que ni un adolescente y joven quede excluido del sistema educativo.

La escuela y el aula se constituyen en una unidad de cambio fundamental (Ferreyra \& Peretti, 2015) para el sujeto y para el contexto. Ello requiere que se articule críticamente tanto el PEI (aspectos formales) como el PCI (aspectos procesuales-prácticos) para dar respuesta a la demanda concreta (De Alba, 1998).

Nuestro estudio muestra también que, aún en el marco del gran esfuerzo que realizan ambas escuelas, persisten aún la repitencia, el abandono escolar y serias dificultades en el aprendizaje de algunos alumnos. Esta pretensión, por tanto, de ser el "último eslabón" en la cadena de segregaciones, antes del abandono, requiere una creciente articulación de la escuela con otros actores, particularmente del estado y de organizaciones de la sociedad civil, que contribuyan a dar formatos de contención.

\footnotetext{
17 La Escuela Virgen Inmaculada comenzó en el 2017 con la modalidad de educación permanente para jóvenes y adultos. Concurren adultos del barrio Ramón Carrillo, entre ellos padres de alumnos de la escuela.
} 
La identificación del perfil de valores interpersonales de estos jóvenes nos permitió reconocer el nivel de valoración intragrupal en las cinco dimensiones propuestas por Gordon. Estos valores interpersonales están relacionados con los comportamientos sociales y se ven afectados por el nivel educativo, la situación laboral del padre o madre. Por ello, al analizar los extremos de la escala obtenida, -Independencia vs. Reconocimiento Social- consideramos fundamental reforzar las intervenciones educativas prosociales articuladas en el PCI y avanzar en una educación en valores y transformación humana y social a mediano y largo plazo tanto de los estudiantes como de sus madres y padres. Los jóvenes entrevistados valoran su escuela y especialmente el aprendizaje como un constructo de alto potencial integrador vinculado a la movilidad social y a las expectativas de construir proyectos de futuro posible, ser alguien para la sociedad y desarrollar sus capacidades cognitivas coincidiendo con trabajos recientemente realizados (Facciola, 2017). Tanto el perfil axiológico como las expectativas derivadas de la valoración del aprendizaje demandan respuestas adecuadas por parte de la escuela.

Uno de los interrogantes presentes en nuestra investigación fue explorar en qué medida las escuelas favorecen al reconocimiento social de los alumnos. Hemos obtenido dos respuestas: una en sentido amplio, la presencia de "la escuela secundaria (en el barrio) valoriza al barrio", pero también en sentido específico, a través de una política educativa vinculada a la política social de las parroquias en los barrios que tiende a posicionar a los jóvenes a partir de la inclusión educativa y social. Una escuela secundaria situada en las periferias existenciales, tanto humana como socialmente, reclama opciones, en primer lugar, que tengan en cuenta la centralidad del sujeto aprendiente, sus necesidades, su perfil axiológico y su contexto y en segunda instancia, que se generen variaciones en el formato escolar (Tiramonti, 2011), capaces de dar respuesta a sus esas realidades.

La motivación manifestada por docentes y directivos es de vital importancia para que los proyectos educativos, se ajusten a las demandas educativas.

A partir de lo presentado, quedan abiertas algunas líneas de investigación; entre ellas: 1 . indagar si se produce un efecto "positivo" en el barrio y cómo en base a la presencia de escuelas en la trama institucional barrial, particularmente si estas escuelas se articulan en torno a un proyecto comunitario; 2 . estudiar nuevos formatos escolares para la escuela secundaria y su posible implementación en estas realidades; 3 . investigar acerca de las diversas experiencias realizadas sobre la base de los currículum pertinentes para diversas contextos socioculturales.

\section{Referencias bibliográficas}

Arocho Rodriguez, W. (2010). El concepto de calidad educativa: Una mirada crítica desde el enfoque sociocultural. Actualidades Investigativas en Educación, 10(1), 1-28.

Bellei, C. (2013). El estudio de segregación socioeconómica y académica de la educación chilena. Revista Estudios Pedagógicos, XXXIX(1), 325-345. 
Bourdieu, P. (1987). Los tres estados del capital cultural. Revista del Departamento de Sociología. Recuperado de http://www.sociologicamexico.azc.uam.mx/index.php/ Sociologica/article/view/1043/1015.

Braslavsky, C. (1985). La discriminación educativa en la Argentina. Buenos Aires: FLACSOGEL.

Briscioli, B. (2015). Tendencias y puntos críticos en las trayectorias escolares de estudiantes de escuelas de reingreso de la Ciudad de Buenos Aires. Una indagación sobre las condiciones de escolarización en la construcción de las trayectorias escolares (Tesis de doctorado inédita). Buenos Aires: Universidad de Buenos Aires.

Carrara, G. (2017). Pasión por correr las fronteras. Capilla, colegio y club, las 3 C. Comunicamos, 17(161), 8-11. Recuperado de https://issuu.com/comunicarnos5/docs/ comunicarnos_julio_agosto_2017.

De Alba, A. (1998). Currículum: crisis, mito y perspectivas. Buenso Aires: Miño y Dávila.

Facciola, M. (2017). Las representaciones sociales del aprendizaje escolar en jóvenes y adultos de Educación Permanente en contextos vulnerables (Tesis de doctorado inédita). Recuperado de http://bibliotecadigital.uca.edu.ar/greenstone/cgi-bin/library.cgi?a $=\mathrm{d} \& \mathrm{c}=$ tesis\&d=representaciones-sociales-aprendizaje-escolar.

Facciola, M. \& Aguilar Rivera, M. (2015). La carpeta escolar: muestrario de actividades y espejo del esfuerzo. Revista de Psicología 23(11), 31-44.

Feito, L. (2007). Vulnerabilidad. Anales del Sistema Sanitario de Navarra, 30(3), 7-22.

Ferreyra, H. \& Peretti, G. (2015). Diseñar y gestionar una educación auténtica. Desarrollo decompetencias en escuelas situadas. Buenos Aires: Noveduc.

Gallart, M.A. (2010). La fragmentación educativa en Argentina. En Programa de las Naciones Unidas para el Desarrollo, Desarrollo humano, inclusión y calidad educativa. Aportes para el desarrollo humano en Argentina (pp. 61-73). Buenos Aires: Autor.

Gasparini, L., Jaume, D. \& Vázquez, E. (2011). La segregación escolar en Argentina. Reconstruyendo la evidencia. CEDLAS. Recuperado de http://cedlas.econo.unlp.edu.ar/ archivos_upload/doc_cedlas123.pdf.

Grace, G. (2007). Misión, mercados y moralidad en las escuelas católicas. Un estudio sociológicos sobre educación católica. Buenos Aires: Aula XXI, Santillana, Educa.

Groisman, F. \& Suárez, A.L. (2009). Urban Segregation in Buenos Aires. En B. Roberts \& R. Wilson (Eds.), Urban Spatial Differentiation and Governance in the Americas (pp. 39-54). Austin: Texas University.

Kaztman, R. \& Queiroz Ribeiro, L. (2008). A Cidade contra a Escola. Segregacao urbana e desigualdades educacionais em grandes cidades da América Latina. Rio de Janeiro: Letra Capital.

Kessler, G. (2002). La experiencia escolar fragmentada. Buenos Aires: IIPE-Unesco.

Krüger, N. (2014). Más allá del acceso: segregación social e inequidad en el sistema educativo argentino. Cuadernos de Economía, 33(63), 513-542. 
Leitao, M. (2010). Memoria de la expresión dramática. Cap. Gisele Barret y la pedagogía de la situación apertura a la dimensión subjetiva y objetiva de realidad pedagógica. Recuperado de https://repositorio.ipcb.pt/bitstream/10400.11/815/1/ML\%20\%20Memorias\%20de\%20expresi\%C3\%B3n\%20dram\%C3\%A1tica.pdf.

Lenon de Villar, O. (2003). Sicología intercultural, equidad y currículum pertinente. Docencia $21,38-47$.

López, N. (2005). Equidad educativa y desigualdad social: Desafíos de la educación en el nuevo escenario latinoamericano. Recuperado de http://unesdoc.unesco.org/images/0014/ 001425/142599s.pdf.

Llosa, S., Sirvent, M.T., Toubes, A. \& Santos, H. (2001). La situación de jóvenes y adultos en Argentina. Revista Brasileira de Educaçao, 8, 22-34.

Montes, N. \& Ziegler, S. (2010). Miradas sobre una experiencia de cambio en la escuela secundaria: nuevos formatos para promover la inclusión educativa. Revista Mexicana de Investigación Educativa, 47, 1075-1092.

Moreno, J. E. (2000). Construcción y adaptación de escalas de medición de valores y virtudes (Informe final de Proyecto de Investigación, FHEyCS). Libertador San Martín, Entre Ríos: Universidad Adventista del Plata.

Niemeyer, B. (2006). El aprendizaje situado: una oportunidad para escapar del enfoque del déficit. Revista de Educación, 341, 99-121. Recuperado de http:// www.revistaeducacion.mec.es/re341/re341_05.pdf.

Nóbile, M. (2014). Redefiniciones del mérito en secundaria: el lugar del esfuerzo en las escuelas de reingreso de la Ciudad de Buenos Aires. CPU-e, Revista de Investigación Educativa, 18, 87-110.

Nóbile, M. (2016). Los egresados de las escuelas de reingreso: sobre los soportes mínimos para aprovechar una política de reinserción educativa. Espacios en blanco. Serie indagaciones, 26, 187-210.

Programa de las Naciones Unidas para el Desarrollo (2010). Desarrollo humano, inclusión y calidad educativa. Aportes para el desarrollo humano en Argentina. Buenos Aires: Autor

Rivas, A. (2010). Tendencias educativas, desigualdades sociales y fragmentación provincial. En Programa de las Naciones Unidas para el Desarrollo, Desarrollo humano, inclusión y calidad educativa. Aportes para el desarrollo humano en Argentina (pp. 23-59). Buenos Aires: Autor.

Rogoff, B. (2012). Aprender sin lecciones: oportunidad para expandir el conocimiento. Revista Infancia y Aprendizaje, 35(2), 233-252.

Rosetti, M. (2014). La segregación escolar como elemento clave en la reproducción de la desigualdad. Santiago de Chile: CEPAL - Naciones Unidas. Recuperado de h t t p : / / www.cepal.org/es/publicaciones/36837-la-segregacion-escolar-como-un-elemento-clavela-reproduccion-la-desigualdad.

Sabatini, F., Cáceres, G. \& Cerda, J. (2004). Segregación residencial en las principales ciudades chilenas: Tendencias de las tres últimas décadas y posibles cursos de acción. Revista EURE, 27, 82. 
Sagástegui, D. (2004). Una apuesta por la cultura: el aprendizaje situado. Sinéctica, 24, 30-39.

Stake, R. E. (2005). Case Studies. En N. K. Denzin \& Y. S. Lincoln (Eds.), Handbook of Qualitative Research (pp. 443-466). California: Sage Pub.

Suárez, A. L. (2015). Creer en las villas. Devociones y prácticas religiosas en los barrios precarios de la Ciudad de Buenos Aires. Buenos Aires: Biblos.

Suárez, A. L., Mitchell, A \& Lépore, E. (2014). Las villas de la Ciudad de Buenos Aires. Territorios frágiles de inclusión social. Buenos Aires: Educa.

Techo Argentina (2013). Relevamiento de asentamientos informales 2013. Buenos Aires: Techo.

Tiramonti, G. (2011). Variaciones sobre la forma escolar. Límites y posibilidades de la escuela media. Rosario: FLACSO-Homo Sapiens Ediciones.

Valenzuela, J.P. (2008). Segregación en el sistema escolar chileno: en la búsqueda de una educación de calidad en un contexto de extrema desigualdad. En Transformaciones del espacio público, II Escuela Chile-Francia (pp. 131-156). Santiago de Chile: Facultad de Arquitectura y Urbanismo, Universidad de Chile.

Veleda, C. (2005). Efectos segregatorios de la oferta educativa. El caso del conurbano bonaerense. CIPPEC, Documento de Trabajo 5.

Wenger, E. (2001). Comunidades de práctica. Aprendizaje, significado e identidad. BarceIona: Paidós. 